Article

\title{
High-Speed Terahertz Waveform Measurement for Intense Terahertz Light Using 100-kHz Yb-Doped Fiber Laser
}

\author{
Masaaki Tsubouchi * (D) and Keisuke Nagashima \\ Kansai Photon Science Institute (KPSI), National Institutes for Quantum and Radiological Science and \\ Technology (QST), 8-1-7 Umimedai, Kizugawa, Kyoto 619-0215, Japan; nagashima.keisuke@qst.go.jp \\ * Correspondence: tsubouchi.masaaki@qst.go.jp; Tel.: +81-774-71-3369
}

Received: 10 May 2018; Accepted: 13 June 2018; Published: 14 June 2018

\begin{abstract}
We demonstrate a high-speed terahertz (THz) waveform measurement system for intense $\mathrm{THz}$ light with a scan rate of $100 \mathrm{~Hz}$. To realize the high scan rate, a loudspeaker vibrating at $50 \mathrm{~Hz}$ is employed to scan the delay time between $\mathrm{THz}$ light and electro-optic sampling light. Because the fast scan system requires a high data sampling rate, we develop an $\mathrm{Yb}$-doped fiber laser with a repetition rate of $100 \mathrm{kHz}$ optimized for effective THz light generation with the output electric field of $1 \mathrm{kV} / \mathrm{cm}$. The present system drastically reduces the measurement time of the $\mathrm{THz}$ waveform from several minutes to $10 \mathrm{~ms}$.
\end{abstract}

Keywords: terahertz; THz spectroscopy; THz imaging; high-speed measurement

\section{Introduction}

Terahertz (THz) light is used for the inspection of semiconductor devices and biological tissue and for security and other applications [1-8]. THz light has low photon energy, which allows nondestructive and noninvasive probes to be used for these inspections without influencing the molecular structure and electronic properties. THz time-domain spectroscopy (THz-TDS) is widely used as a sensitive and accurate method to obtain both $\mathrm{THz}$ waveforms and its Fourier transform spectra of THz light. Using this advantage of THz-TDS, a time-of-flight THz tomography has been developed for measurement of layer structures near the surface [9]. In addition, a two-dimensional (2D) THz hyperspectral imaging based on THz-TDS has been demonstrated for the inspection of drugs and food items, for investigation of ancient arts, and for medical diagnostics [10-13] and has been commercially available.

To establish $\mathrm{THz}$ imaging as a practical tool, the acquisition speed of $\mathrm{THz}$ imaging systems with THz-TDS must be increased by two to three orders of magnitude, as suggested in the roadmap of the $\mathrm{THz}$ science and technology given in Reference [14]. The slow acquisition speed of $\mathrm{THz}$ hyperspectral imaging is mainly because the time-domain scan needs to obtain a waveform in THz-TDS in addition to the spatial 2D scan. In the conventional THz-TDS setup, a linear mechanical delay stage is employed to scan the time delay between the $\mathrm{THz}$ and the sampling pulses. Because the stage is decelerated and accelerated in each measuring step of the $\mathrm{THz}$ electric field, the acquisition time of the entire $\mathrm{THz}$ waveform easily exceeds several minutes. Therefore, we have to establish a method of high-speed $\mathrm{THz}$ waveform measurement for practical $\mathrm{THz}$ imaging.

In THz-TDS systems that use weak femtosecond pump light with repetition rates of more than $10 \mathrm{MHz}$, high-speed THz waveform measurements have already been demonstrated. The mechanically rotating mirrors have realized scan rates of $400 \mathrm{~Hz}[15,16]$. Some studies have proposed electro-optical control of time delay, rather than the mechanical delay scan, for high-speed measurements with $\mathrm{kHz}$ 
scan rates. These include asynchronous optical sampling [17,18], optical sampling by cavity tuning [19], and electrically controlled optical sampling [20,21]. Two mode-locked lasers with a fixed difference of repetition rates introduce a linear sweep of time-delay between the pump and probe pulses. The scan rate of the $\mathrm{THz}$ waveform is equal to the difference of the repetition rates, which is in the range of 0.1 to $10 \mathrm{kHz}$.

Intense $\mathrm{THz}$ light has been obtained in table-top laser systems. However, the low repetition rate of the amplified laser system limits the scan rate to below $1 \mathrm{~Hz}$. A high scan rate in THz-TDS with intense $\mathrm{THz}$ light can extend $\mathrm{THz}$ applications. Photo-induced dynamics of semiconductor, polymer, biological tissue, etc. can be effectively measured by optical pump-THz probe time-resolved spectroscopy with high-speed measurements. The THz imaging system ensures that high speed simultaneous $\mathrm{THz}$ waveform measurements in $1 \mathrm{D}$ or 2D space are feasible with the intense $\mathrm{THz}$ light. To realize high-speed $\mathrm{THz}$ waveform measurements with intense $\mathrm{THz}$ light, single-shot measurements of the waveform without time-domain scan have been proposed. These are based on mapping of the time-domain data onto the spatial position of the electro-optic (EO) crystal by noncollinear geometry for the $\mathrm{THz}$ and probe light [22] or a reflective echelon mirror [23], and mapping onto the frequency spectrum by a chirped probe pulse $[24,25]$. The scan rate of these promising methods depends on the laser's repetition rate and the data accumulation time and is typically 1 to $10 \mathrm{~Hz}$.

In this study, we have developed an advanced method to achieve high-speed measurement of the $\mathrm{THz}$ waveform with intense $\mathrm{THz}$ light. Our method is based on the time-domain scan with high-speed vibration of a translational stage and achieves a scan rate of $100 \mathrm{~Hz}$ with easy operation. In previous single-shot measurements, the waveform had to be transformed from the observed spatial mapping or spectrum with appropriate calibration. The parameters used in the calibration are sensitive to the optical alignment and, therefore, must be determined in each experiment. Furthermore, it is difficult to linearly map the time-domain data onto the spatial or spectral region across a wide range (typically less than $10 \mathrm{ps}$ ). By contrast, our method does not require complicated calibration because the $\mathrm{THz}$ waveform is directly measured by the time-domain scan. The time range of the scan is only limited by the maximum vibration amplitude of the translation stage.

This paper is organized as follows: First, we describe experimental details of high-speed $\mathrm{THz}$ waveform measurement in Section 2. To realize the high-speed measurement of the $\mathrm{THz}$ waveform with intense $\mathrm{THz}$ light and to apply it to practical study such as the $\mathrm{THz}$ hyperspectral imaging, we thoroughly design the total system and optimize well-known technical components, e.g., an Yb-doped fiber laser, a loudspeaker scanner, a THz-TDS system, and so on. Next, we demonstrate and evaluate our system in Section 3. We conclude with future perspectives in Section 4.

\section{Experimental Details}

We constructed a system based on a high-speed time-domain scan to obtain $\mathrm{THz}$ waveforms with a high scan rate. To scan a time delay between the THz and EO probe pulses quickly, we used a loudspeaker as a high-speed scanner that has been used in autocorrelators to measure the pulse width of ultrashort laser pulses [26]. This method has been introduced for $\mathrm{THz}$ waveform measurement in a photoconductive antenna system [27]. In our system, the achieved scan rate of the waveform was $100 \mathrm{~Hz}$ with the speaker's vibration at $50 \mathrm{~Hz}$; two waveforms were obtained in a single round trip. The mechanically rotating mirror was also one of the candidates for the high-speed time-domain scan for intense THz light. However, when we used the rotating mirror to reflect the intense pump light, we had to process a high-reflection (HR) multilayer coating on the curved metal surface of the rotating mirror, which was technically very difficult. On the other hand, we could use the bare metal surface as the rotating mirror for the weak EO probe light. But the rotating mirror was not proper for the scan of the probe light because EO detection is very sensitive to the beam pointing of the probe light on the EO crystal. As a result, we adopted the loudspeaker as a high-speed scanner instead of the rotating mirror.

When the scanning system is operated at $100 \mathrm{~Hz}$, the repetition rate of $\mathrm{THz}$ pulse generation has to be increased also. For example, the $\mathrm{THz}$ generation system with a $1 \mathrm{kHz}$ repetition rate can plot 
only 10 points in a single waveform, which is insufficient for practical $\mathrm{THz}$ waveform measurement. Therefore, we constructed a laser system with a $100 \mathrm{kHz}$ repetition rate in which 1000 data points can be plotted in a waveform.

\subsection{THz Light Generation}

First, we designed the effective $\mathrm{THz}$ generation scheme with a $100 \mathrm{kHz}$ repetition rate. Currently, an optical rectification in $\mathrm{LiNbO}_{3}(\mathrm{LN})$ crystal with a titled pulse front technique is widely used to generate intense THz light [28-30]. Fülöp and co-workers calculated the THz generation process in this method and suggested that the $\mathrm{THz}$ generation efficiency was maximized for the transform-limited (TL) pump pulse at the pulse width of 300-400 fs [31]. The Yb-doped fiber laser is one of the most promising systems for effective $\mathrm{THz}$ light generation with a $350 \mathrm{fs}$ pulse-width and a $100 \mathrm{kHz}$ repetition rate. Figure 1 shows the schematic of the $\mathrm{Yb}$-doped fiber laser system constructed in this study. A mode-locked fiber oscillator operated at a center wavelength of $1040 \mathrm{~nm}$ and a frequency of $43 \mathrm{MHz}$ was employed as a seed laser. The stretched seed laser was amplified to $1 \mathrm{~W}$ in two Yb-doped fibers pumped by a 976-nm laser diode. After the second amplifier, $100 \mathrm{kHz}$ pulses with $1 \mathrm{~mW}$ were picked from $43 \mathrm{MHz}$ by a Pockels cell and amplified again in four fiber amplifiers to $5 \mathrm{~W}$. The amplified $100 \mathrm{kHz}$ pulses were sent to the pulse compressor and compressed to the TL pulses with a pulse energy of $28 \mu \mathrm{J}$ and a pulse width of $320 \mathrm{fs}$ for $\mathrm{THz}$ light generation. As mentioned before, the amplification process was divided into six stages to suppress amplified spontaneous emission and to prevent damage to the fibers. The amplified laser power after the compressor increased linearly with increasing diode current in the final amplifier, reaching a maximum value of $10 \mathrm{~W}(100 \mu \mathrm{J} / \mathrm{pulse})$. However, when the output energy was larger than $28 \mu \mathrm{J}$, the amplified pulse could not be compressed to the TL pulse at the compressor due to higher-order dispersion caused by self-phase modulation.

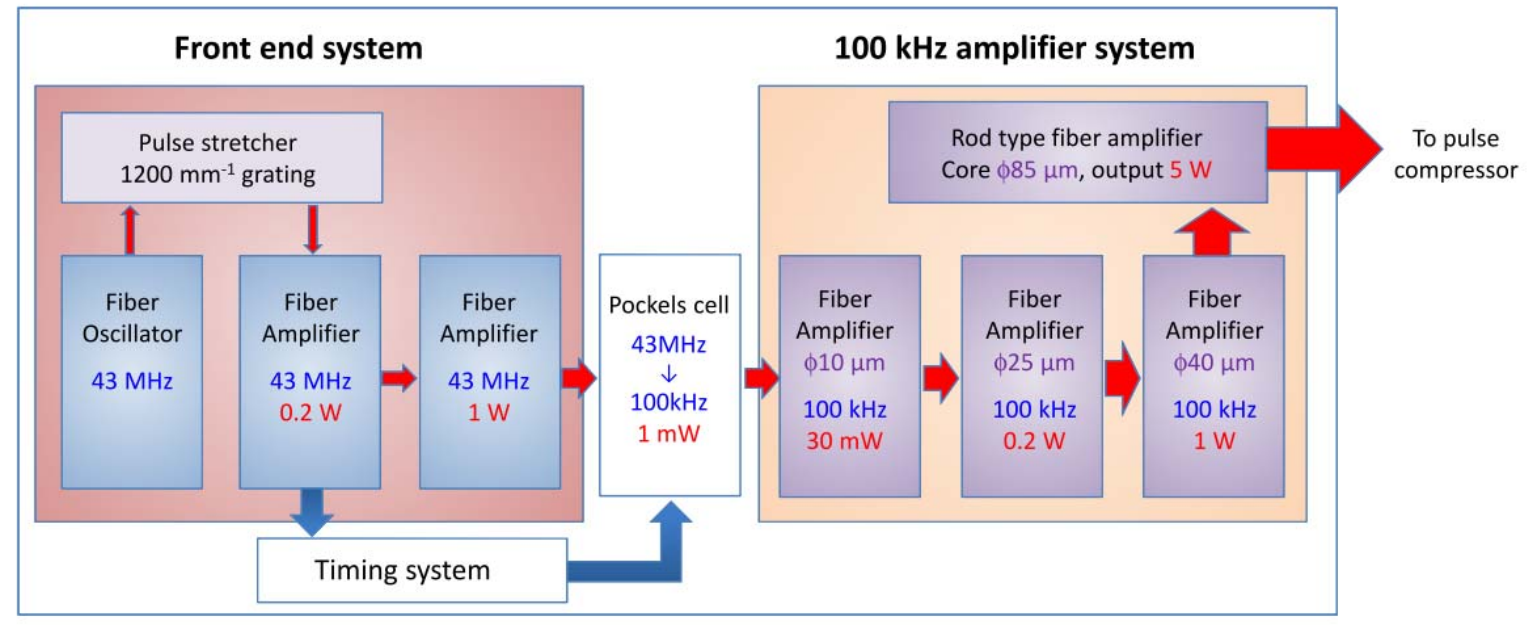

Figure 1. Schematic of the Yb-doped fiber laser system.

The near-infrared (NIR) pump light was delivered to the THz generation system based on the "contact grating setup", which was first proposed by Pálfalvi and co-workers [32] and was realized in our previous studies [33-35]. In the contact grating setup, the diffraction grating was placed in contact with the input surface of the LN substrate, which resulted in a drastic downsizing of the THz generation system compared with the conventional pulse front tilting method. Therefore, the generation system with the contact grating setup is promising for industrial applications. The contact grating device was fabricated on a $1.3 \mathrm{moL} \% \mathrm{Mg}$-doped stoichiometric LN (Mg-sLN) substrate with dimensions $16 \mathrm{~mm}(\mathrm{Z})$ $\times 20 \mathrm{~mm}(\mathrm{Y}) \times 2.2 \mathrm{~mm}(\mathrm{X})$, where $X, Y$, and $Z$ are the crystal axes. The grooves were etched parallel to the $Z$-axis with an effective area of $10 \mathrm{~mm}(\mathrm{Z}) \times 14 \mathrm{~mm}(\mathrm{Y})$. Details of the device are described in our previous paper [33-35]. 


\subsection{THz Waveform Measurement}

Figure 2 shows the schematic diagram of the $\mathrm{THz}$ generation and detection system. The output NIR pulse from the $\mathrm{Yb}$-doped fiber laser was split into two parts for $\mathrm{THz}$ light generation and EO detection. The former part was passed through the polarizing beam splitter (PBS) and reflected by the mirror installed on the vibrating membrane of the speaker. To pick up the reflected light by PBS, the quarter wave plate was placed between PBS and the speaker. The reflected light pumps the contact grating device to generate $\mathrm{THz}$ light. Both the pump and $\mathrm{THz}$ light were s-polarized to the device. The THz light was separated from the pump light by a plastic plate with a HR coating for the NIR light and a black polypropylene film. The THz light was collimated by an aspheric plastic lens and focused to a detection point by a gold-coated off-axis parabolic mirror. At the detection point, the $\mathrm{THz}$ electric field was observed by EO sampling in a 3-mm-thick CdTe crystal. The EO probe light with vertical polarization was reflected by the mirror pair on the stepping motor translation stage and focused on the CdTe crystal. The polarization rotation due to the EO effect was measured by a balanced detection system with a balanced amplified photodetector.

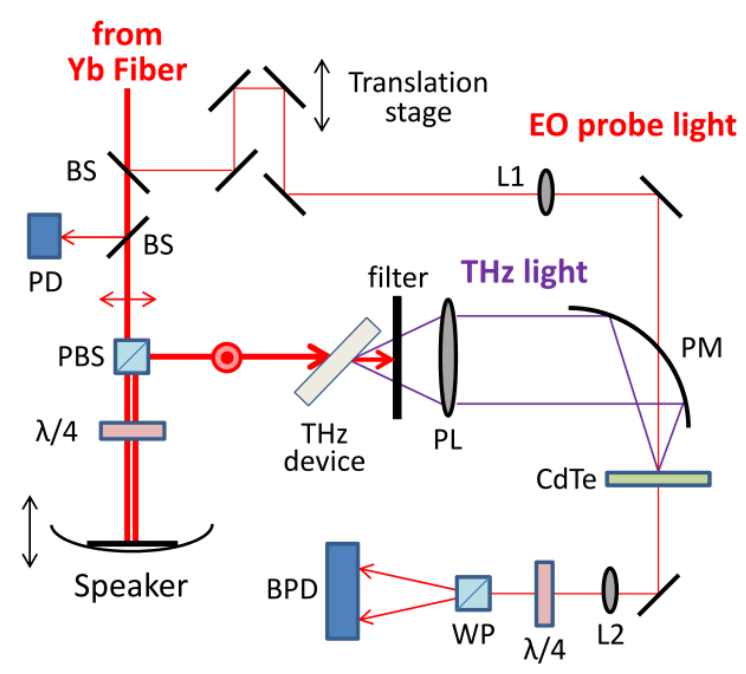

Figure 2. Schematic diagram of the $\mathrm{THz}$ generation and detection system. BS: beam splitter; PD: fast photodiode for 100-kHz laser trigger; PBS: polarizing beam splitter; $\lambda / 4$ : quarter wave plate; L1 and L2: BK7 lenses with focal lengths of 250 and $150 \mathrm{~mm}$, respectively; PL: plastic lens for THz light with a focal length of $50 \mathrm{~mm}$; PM: off-axis parabolic gold-coated mirror with a centered hole $2 \mathrm{~mm}$ in diameter and a focal length of $50 \mathrm{~mm}$; WP: Wollaston prism; BPD: balanced amplified photodetector.

The optical delay between the $\mathrm{THz}$ and EO probe light was first adjusted by the stepping motor translational stage and then scanned by the speaker's vibration. The appropriate speaker was chosen so that the amplitude of the membrane motion ensures a linear with respect to an input voltage. Figure 3 shows the block diagram of the data acquisition system. The Yb-fiber laser generated the $100 \mathrm{kHz}$ clock to the system. The analog EO signal from the balanced detector was recorded via a data acquisition (DAQ) device synchronized with the $100-\mathrm{kHz}$ laser trigger. The speaker vibration was controlled by a function generator, which provided a triangular wave at frequency of $50 \mathrm{~Hz}$. The measurement of the $\mathrm{THz}$ waveforms was triggered by $50 \mathrm{~Hz}$ TTL signal from the function generator and achieved a scan rate of $100 \mathrm{~Hz}$ as mentioned before. 


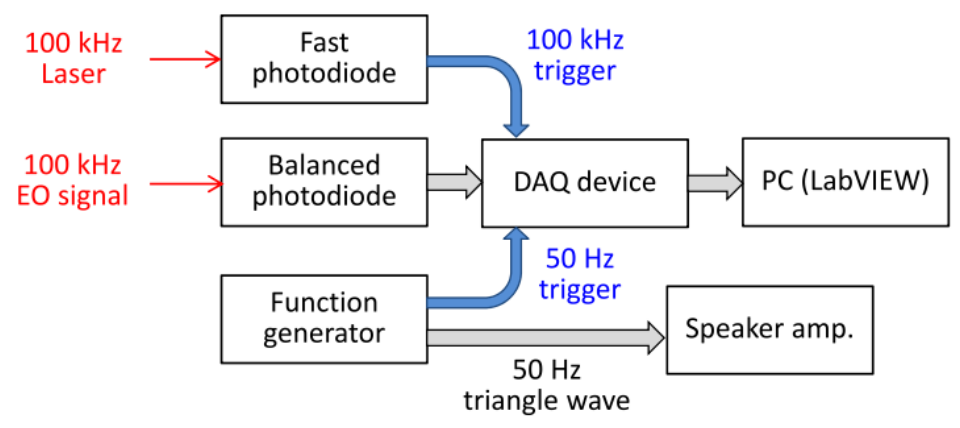

Figure 3. Block diagram of the data acquisition system.

\section{Results and Discussion}

Figure 4 shows the $\mathrm{THz}$ waveforms obtained by the data acquisition system described in Figures 2 and 3. The horizontal axis shows the time at which the data was recorded by the DAQ device. The sequence of the $\mathrm{THz}$ waveforms was successfully obtained without averaging. Data acquisition was synchronized with a $100-\mathrm{kHz}$ laser trigger and, therefore, the data sampling interval in the waveform was $10 \mu \mathrm{s}$. The triangular wave applied to the speaker system is indicated in the Figure 4 as the blue dashed line. The stepping motor translation stage was adjusted so that the THz electric field took the maximum value at the zero-vibration amplitude of the speaker. When a positive voltage was applied to the speaker, the EO probe pulse arrived before the pump pulse at the EO sampling point and vice versa. Within the single period of the triangular wave, a pair of $\mathrm{THz}$ waveforms was observed.

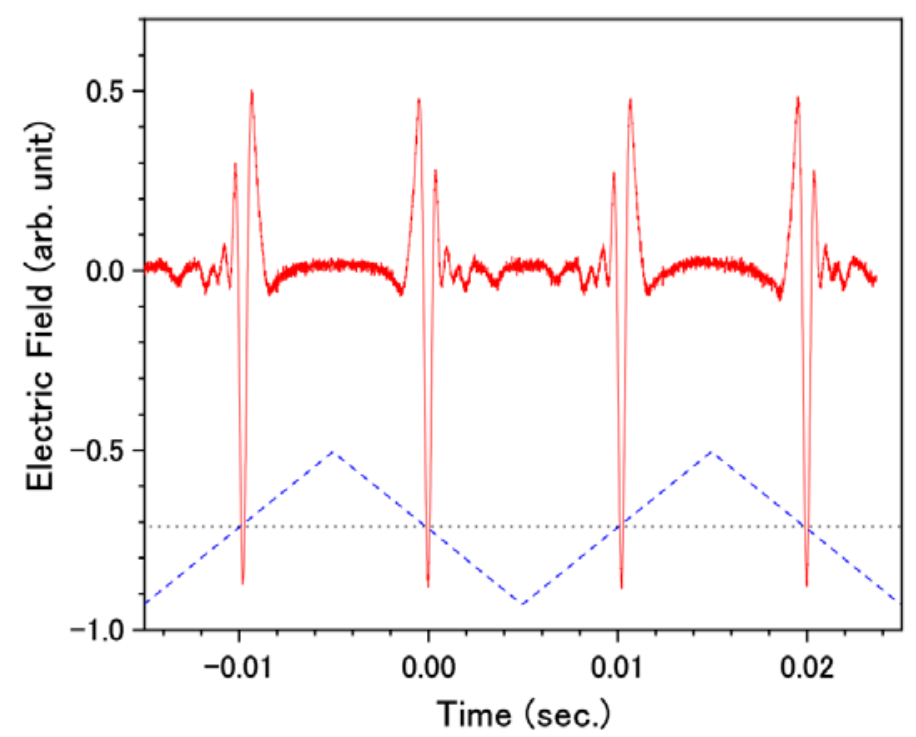

Figure 4. THz waveforms obtained by the data acquisition system described in Figures 2 and 3. The horizontal axis shows the time at which the data was recorded by data acquisition (DAQ) device. The red solid line shows the observed waveform. The blue dashed line indicates the vibration of the speaker membrane around the zero-time delay between the electro-optic (EO) probe and $\mathrm{THz}$ light (shown as the black dotted line).

To calibrate the delay time of the EO probe pulse from the pump pulse, we measured the $\mathrm{THz}$ waveforms at several positions of the stepping motor translation stage as shown in Figure 5. Three series of the $\mathrm{THz}$ waveform-A, B, and C-are shown in Figure 5. The time interval between series $A$ and $C$ was always $20 \mathrm{~ms}$, which was identical to the period of the speaker's vibration. The time when series A appeared, $t_{D A Q}$, linearly shifted to the later time when increasing the position of the translational stage, $X: t_{D A Q}=a X$, where $a$ is the proportionality constant and we define as $t_{D A Q}=0$ 
at $X=0$. This result indicates that the displacement of the speaker membrane is proportional to the amplitude of the triangular wave. The delay time $t$ of the EO probe pulse from the pump pulse was related to the position of the translational stage at the specific $t_{D A Q}$ as $t=2 X / c_{0}$, where $c_{0}$ is the speed of light. Therefore, the delay time $t$ can be calibrated as $t=2 t_{D A Q} / a c_{0}$. In Figure $6 \mathrm{a}$, the calibrated waveform is shown as the solid line and is compared with the waveform measured by the stepping motor translation stage. There is good agreement between waveforms. The $\mathrm{THz}$ waveform consists of 1000 data points in the time range of 15 ps with $15 \mathrm{fs}$ intervals. The single waveform with the time range of 15 ps was measured in the measurement time of $10 \mathrm{~ms}$, which was $10^{4}$ times shorter than that required for the scan by the stepping motor translation stage. By employing pump light with a pulse energy of $13 \mu \mathrm{J}$, the maximum THz electric field of $0.96 \mathrm{kV} / \mathrm{cm}$ was obtained. Figure $6 \mathrm{~b}$ shows the Fourier transformed spectra. The peak frequency and the bandwidth were $0.6 \mathrm{THz}$ and $1.5 \mathrm{THz}$, respectively. It can be noticed that the waveforms are slightly distorted near the turning points of the membrane vibration in the loudspeaker, as seen in Figure 5a,e. This is due to the lack of linearity in the amplitude of the membrane motion with respect to the applied voltage at the turning points. To understand the distortion of the waveforms quantitatively, we calculated the Fourier transformed spectra from the waveforms shown in Figure 5a-e and compared these spectra with the reference spectrum obtained by conventional translational stage. The spectrum (c) in which the THz electric field took the maximum value at the zero-vibration amplitude of the speaker was almost identical to the reference. However, in the spectra (a) and (e), the discrepancy due to the lack of linearity in the membrane motion can be seen in the high and low frequency regions.

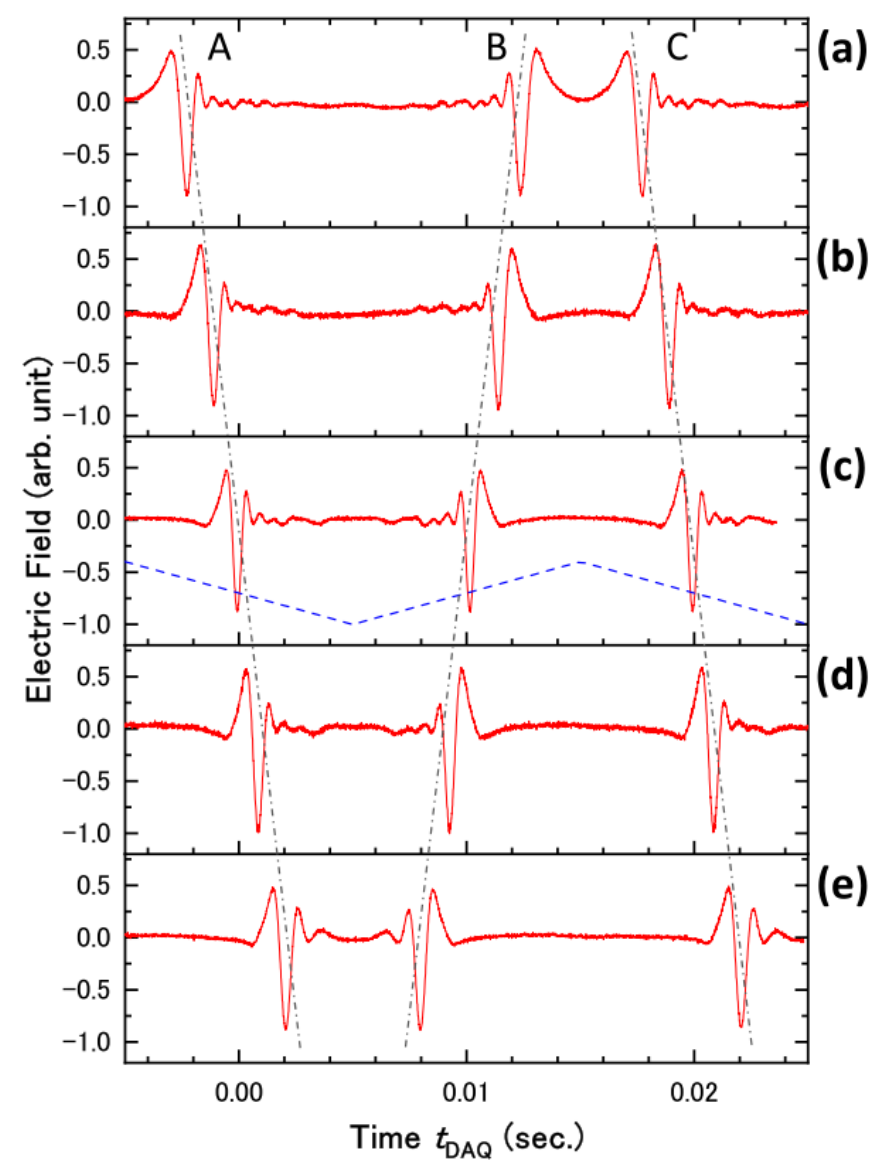

Figure 5. THz waveform observed at the position of the stepping motor translational stage, (a) $X=-500 \mu \mathrm{m}$; (b) $-250 \mu \mathrm{m}$; (c) $0 \mu \mathrm{m}$; (d) $250 \mu \mathrm{m}$; and (e) $500 \mu \mathrm{m}$. The red solid line shows the observed waveform. The blue dashed line indicates the vibration of the speaker membrane. 


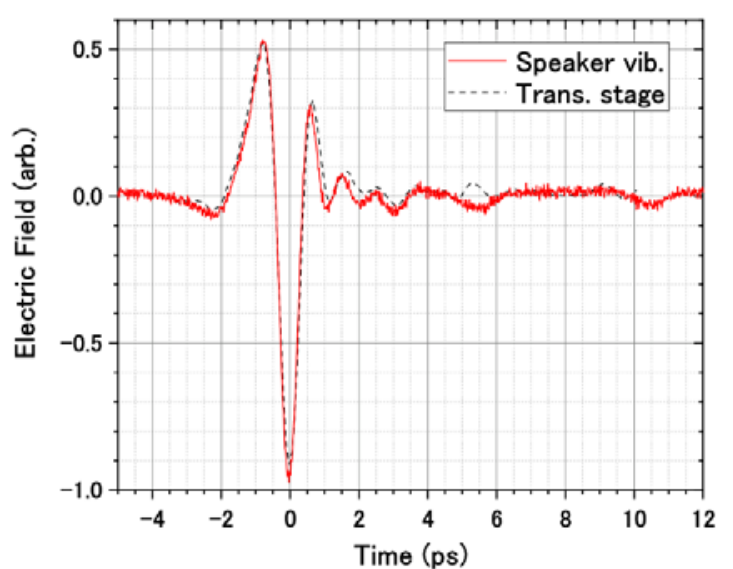

(a)

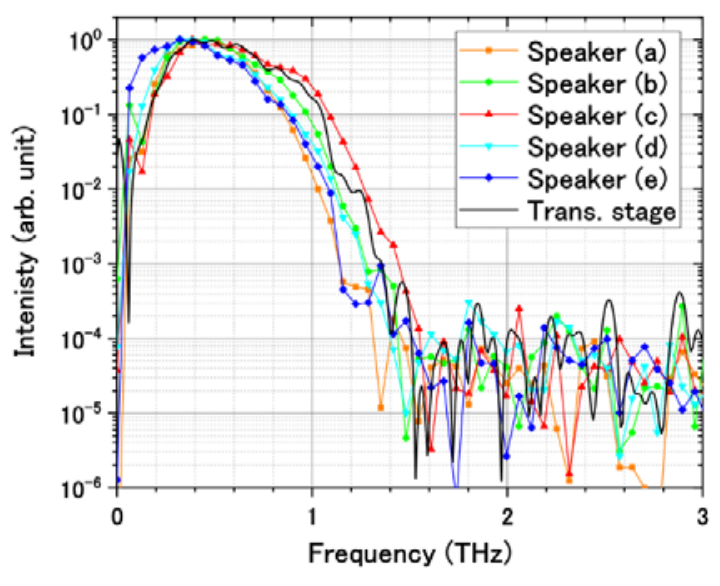

(b)

Figure 6. (a) THz waveforms and (b) its Fourier transformed spectra obtained by using the stepping motor translational stage and the vibrating speaker. The "speaker (a) to (e)" indicates the spectrum obtained from the waveform shown in Figure 5a-e, respectively.

In the presented study, we have several important parameters describing the apparatus: the scan rate, the frequency resolution, and the signal-to-noise ratio (SNR). The frequency resolution depends on the scanning time range, which is closely related to the scan rate. In our system, we supplied the triangular wave with a frequency of $50 \mathrm{~Hz}$ to the speaker and scanned the waveform in the time range of $15 \mathrm{ps}$. In principle, the scan rate of the $\mathrm{THz}$ waveform increases with increasing vibration frequency of the speaker. However, the allowed maximum vibration amplitude of the speaker at high frequencies is too small to scan enough time range in the THz waveform. This mechanical threshold for the speaker limits the scan rate of the waveforms. To discuss the SNR in our apparatus, Figure $7 \mathrm{a}, \mathrm{b}$ show the $\mathrm{THz}$ waveforms and the Fourier transformed spectra measured by changing the cumulative number, respectively. The SNR of the spectrum was $10^{4}$ even without averaging and went up to $10^{6}$ with $1 \mathrm{~s}$ (100 scans) averaging. As shown in Figure 7, our system realizes the high-precision measurement of the $\mathrm{THz}$ waveform within $1 \mathrm{~s}$, which drastically reduces the data acquisition time for the practical applications of the THz-TDS with intense THz light, for example, optical pump-THz probe experiments, time-of-flight tomography, and $\mathrm{THz}$ imaging. 


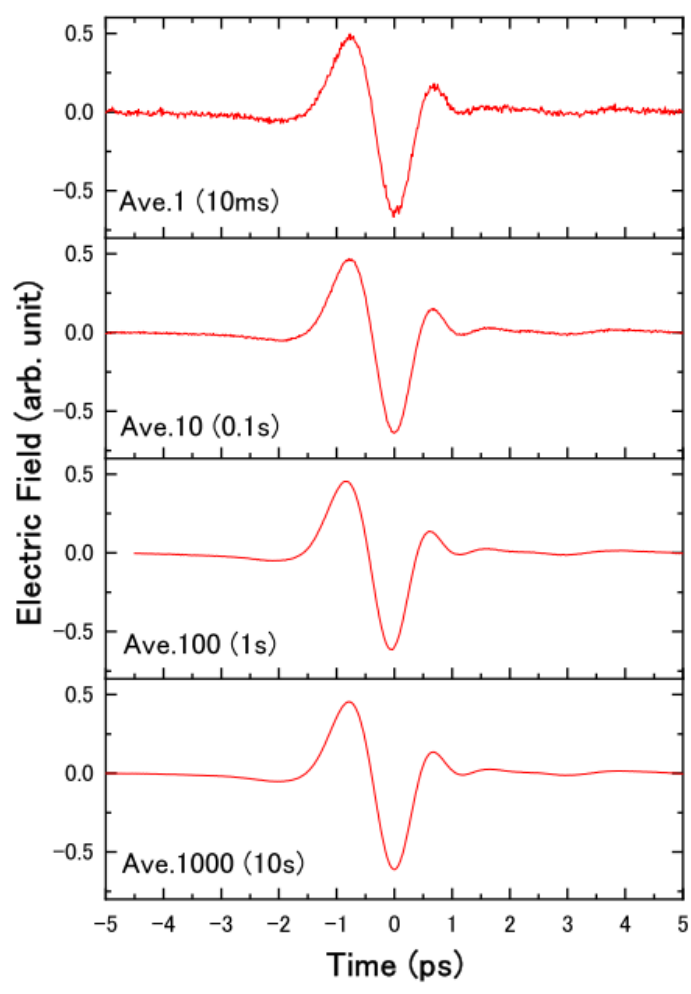

(a)

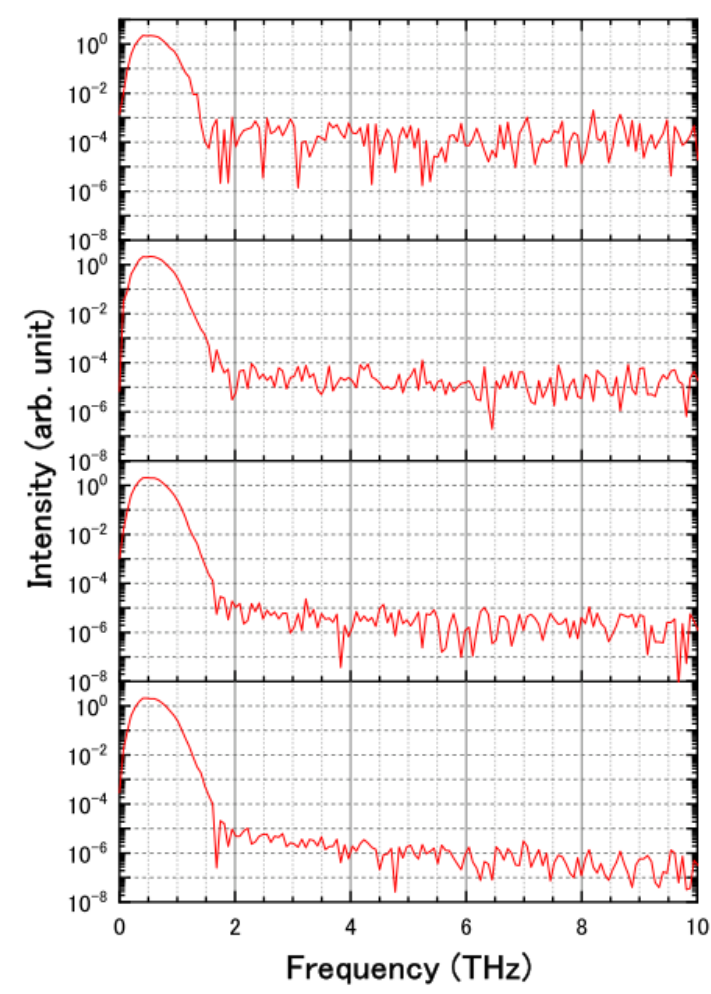

(b)

Figure 7. (a) $\mathrm{THz}$ waveforms and (b) Fourier transformed spectra measured by changing the cumulative number.

\section{Summary and Perspectives}

In this study, high-speed $\mathrm{THz}$ waveform measurement with intense $\mathrm{THz}$ light has been demonstrated with a scan rate of $100 \mathrm{~Hz}$. To obtain high-speed measurement, we employed a loudspeaker as the high-speed scanner for the delay time of the EO probe light with respect to the $\mathrm{THz}$ light, a 100-kHz Yb-doped fiber laser system for high-speed data acquisition, and a contact grating device for $\mathrm{THz}$ light generation.

One of the advantages of THz-TDS is that the carrier-envelope phase-locked few-cycle $\mathrm{THz}$ pulse is observed directly. Therefore, it is easily applied to time-resolved spectroscopy (e.g., optical pump- $\mathrm{THz}$ probe spectroscopy), time-of-flight $\mathrm{THz}$ tomography, and $\mathrm{THz}$ hyperspectral imaging. The hyperspectra include the spectral information in each pixel distributed in 2D space. Therefore, 3D time-space scanning is required in the conventional THz-TDS with weak THz light. When we apply our fast measurement system with intense $\mathrm{THz}$ light, we measure the spectra in pixels of 1D line or 2D plane simultaneously. This is expected to drastically reduce the measurement time of the hyperspectral image from $1 \mathrm{~h}$ to $1 \mathrm{~s}$. Our high-speed $\mathrm{THz}$ waveform measurement system will accelerate these applications greatly and promote the industrial application of THz science.

Author Contributions: M.T. wrote the paper; all authors contributed edits to the paper; M.T. contributed to development of the THz generation and waveform measurement; K.N. contributed to development of Yb-doped fiber laser system and design of the THz generation device.

Funding: This research was funded by the Japan Science and Technology Agency under Collaborative Research Based on Industrial Demand “Terahertz-wave: Towards Innovative Development of Terahertz-wave Technologies", and the CPhoST program funded by the Special Coordination Funds for Promoting Science and Technology commissioned by the Ministry of Education, Science, Culture, and Sports of Japan.

Conflicts of Interest: The authors declare no conflict of interest. 


\section{References}

1. Han, P.Y.; Zhang, X.C. Free-space coherent broadband terahertz time-domain spectroscopy. Meas. Sci. Technol. 2001, 12, 1747-1756. [CrossRef]

2. Beard, M.C.; Turner, G.M.; Schmuttenmaer, C.A. Terahertz spectroscopy. J. Phys. Chem. B 2002, 106, 7146-7159. [CrossRef]

3. Ferguson, B.; Zhang, X.C. Materials for terahertz science and technology. Nat. Mater. 2002, 1, 26-33. [CrossRef] [PubMed]

4. Schmuttenmaer, C.A. Exploring dynamics in the far-infrared with terahertz spectroscopy. Chem. Rev. 2004, 104, 1759-1779. [CrossRef] [PubMed]

5. Jepsen, P.U.; Cooke, D.G.; Koch, M. Terahertz spectroscopy and imaging-Modern techniques and applications. Laser Photonics Rev. 2011, 5, 124-166. [CrossRef]

6. Lee, Y.-S. Principles of Terahertz Science and Technology; Springer: New York, NY, USA, 2009.

7. Son, J.-H. Terahertz Biomedical Science and Technology; CRC Press: Boca Raton, FL, USA, 2014.

8. Song, H.-J.; Nagatsuma, T. Handbook of Terahertz Technologies Devices and Applications; Pan Stanford Publishing: Singapore, 2015.

9. Zhang, X.C. Three-dimensional terahertz wave imaging. Philos. Trans. R. Soc. Lond. A 2004, 362, $283-298$. [CrossRef] [PubMed]

10. Kawase, K.; Ogawa, Y.; Watanabe, Y.; Inoue, H. Non-destructive terahertz imaging of illicit drugs using spectral fingerprints. Opt. Express 2003, 11, 2549-2554. [CrossRef] [PubMed]

11. Guillet, J.P.; Recur, B.; Frederique, L.; Bousquet, B.; Canioni, L.; Manek-Honninger, I.; Desbarats, P.; Mounaix, P. Review of terahertz tomography techniques. J. Infrared Millim. Terahertz Waves 2014, 35, 382-411. [CrossRef]

12. Abraham, E.; Fukunaga, K. Terahertz imaging applied to the examination of artistic objects. Stud. Conserv. 2015, 60, 343-352. [CrossRef]

13. Sun, Q.S.; He, Y.Z.; Liu, K.; Fan, S.T.; Parrott, E.P.J.; Pickwell-MacPherson, E. Recent advances in terahertz technology for biomedical applications. Quant. Imaging Med. Surg. 2017, 7, 345-355. [CrossRef] [PubMed]

14. Dhillon, S.S.; Vitiello, M.S.; Linfield, E.H.; Davies, A.G.; Hoffmann, M.C.; Booske, J.; Paoloni, C.; Gensch, M.; Weightman, P.; Williams, G.P.; et al. The 2017 terahertz science and technology roadmap. J. Phys. D Appl. Phys. 2017, 50, 043001. [CrossRef]

15. Xu, J.Z.; Zhang, X.C. Circular involute stage. Opt. Lett. 2004, 29, 2082-2084. [CrossRef] [PubMed]

16. Kim, G.J.; Jeon, S.G.; Kim, J.I.; Jin, Y.S. Terahertz pulse detection using rotary optical delay line. Jpn. J. Appl. Phys. 2007, 46, 7332-7335. [CrossRef]

17. Bartels, A.; Cerna, R.; Kistner, C.; Thoma, A.; Hudert, F.; Janke, C.; Dekorsy, T. Ultrafast time-domain spectroscopy based on high-speed asynchronous optical sampling. Rev. Sci. Instrum. 2007, 78, 035107. [CrossRef] [PubMed]

18. Klatt, G.; Gebs, R.; Janke, C.; Dekorsy, T.; Bartels, A. Rapid-scanning terahertz precision spectrometer with more than 6 thz spectral coverage. Opt. Express 2009, 17, 22847-22854. [CrossRef] [PubMed]

19. Wilk, R.; Hochrein, T.; Koch, M.; Mei, M.; Holzwarth, R. Terahertz spectrometer operation by laser repetition frequency tuning. J. Opt. Soc. Am. B Opt. Phys. 2011, 28, 592-595. [CrossRef]

20. Kim, Y.; Yee, D.S. High-speed terahertz time-domain spectroscopy based on electronically controlled optical sampling. Opt. Lett. 2010, 35, 3715-3717. [CrossRef] [PubMed]

21. Dietz, R.J.B.; Vieweg, N.; Puppe, T.; Zach, A.; Globisch, B.; Gobel, T.; Leisching, P.; Schell, M. All fiber-coupled thz-tds system with khz measurement rate based on electronically controlled optical sampling. Opt. Lett. 2014, 39, 6482-6485. [CrossRef] [PubMed]

22. Shan, J.; Weling, A.S.; Knoesel, E.; Bartels, L.; Bonn, M.; Nahata, A.; Reider, G.A.; Heinz, T.F. Single-shot measurement of terahertz electromagnetic pulses by use of electro-optic sampling. Opt. Lett. 2000, 25, 426-428. [CrossRef] [PubMed]

23. Minami, Y.; Hayashi, Y.; Takeda, J.; Katayama, I. Single-shot measurement of a terahertz electric-field waveform using a reflective echelon mirror. Appl. Phys. Lett. 2013, 103, 051103. [CrossRef]

24. Jiang, Z.P.; Zhang, X.C. Single-shot measurement of a terahertz pulse. Appl. Opt. 1998, 37, 8145-8146. [CrossRef] 
25. Van Tilborg, J.; Schroeder, C.B.; Toth, C.; Geddes, C.G.R.; Esarey, E.; Leemans, W.P. Single-shot spatiotemporal measurements of high-field terahertz pulses. Opt. Lett. 2007, 32, 313-315. [CrossRef] [PubMed]

26. Kalpaxis, A.; Doukas, A.G.; Budansky, Y.; Rosen, D.L.; Katz, A.; Alfano, R.R. Description of an electronic controller used with an autocorrelator to measure a femtosecond pulse duration in real-time. Rev. Sci. Instrum. 1982, 53, 960-962. [CrossRef]

27. Jin, Y.S.; Jeon, S.G.; Kim, G.J.; Kim, J.I.; Shon, C.H. Fast scanning of a pulsed terahertz signal using an oscillating optical delay line. Rev. Sci. Instrum. 2007, 78, 023101. [CrossRef] [PubMed]

28. Hebling, J.; Almási, G.; Kozma, I.Z.; Kuhl, J. Velocity matching by pulse front tilting for large-area thz-pulse generation. Opt. Express 2002, 10, 1161-1166. [CrossRef] [PubMed]

29. Stepanov, A.G.; Hebling, J.; Kuhl, J. Efficient generation of subpicosecond terahertz radiation by phase-matched optical rectification using ultrashort laser pulses with tilted pulse fronts. Appl. Phys. Lett. 2003, 83, 3000-3002. [CrossRef]

30. Stepanov, A.G.; Kuhl, J.; Kozma, I.Z.; Riedle, E.; Almasi, G.; Hebling, J. Scaling up the energy of thz pulses created by optical rectification. Opt. Express 2005, 13, 5762-5768. [CrossRef] [PubMed]

31. Fülöp, J.A.; Pálfalvi, L.; Almási, G.; Hebling, J. Design of high-energy terahertz sources based on optical rectification. Opt. Express 2010, 18, 12311-12327. [CrossRef] [PubMed]

32. Palfalvi, L.; Fulop, J.A.; Almasi, G.; Hebling, J. Novel setups for extremely high power single-cycle terahertz pulse generation by optical rectification. Appl. Phys. Lett. 2008, 92, 171107. [CrossRef]

33. Tsubouchi, M.; Nagashima, K.; Yoshida, F.; Ochi, Y.; Maruyama, M. Contact grating device with fabry-perot resonator for effective terahertz light generation. Opt. Lett. 2014, 39, 5439-5442. [CrossRef] [PubMed]

34. Yoshida, F.; Nagashima, K.; Tsubouchi, M.; Ochi, Y.; Maruyama, M.; Sugiyama, A. High-efficiency contact grating fabricated on the basis of a fabry-perot type resonator for terahertz wave generation. Jpn. J. Appl. Phys. 2016, 55, 012201. [CrossRef]

35. Nagashima, K.; Tsubouchi, M.; Ochi, Y.; Maruyama, M. Improvement of contact grating device for efficient terahertz wave generation using bi-angular filter. J. Appl. Phys. 2018, 123, 123104. [CrossRef]

(C) 2018 by the authors. Licensee MDPI, Basel, Switzerland. This article is an open access article distributed under the terms and conditions of the Creative Commons Attribution (CC BY) license (http:/ / creativecommons.org/licenses/by/4.0/). 